\title{
GENERALIZATION OF SOME INEQUALITIES VIA RIEMANN-LIOUVILLE FRACTIONAL CALCULUS
}

\author{
MARCELA V. MIHAI AND DANIEL ALEXANDRU ION
}

\begin{abstract}
Some Hermite-Hadamard type inequalities are provided. We deal with functions whose derivatives in absolute value are convex or concave. By defining two cumulative gaps which enable us to generalize known results in the framework of RiemannLiouville fractional calculus, we open a new perspective on the classic statement of the inequality.
\end{abstract}

\section{Introduction}

The Hermite-Hadamard inequality states that if a function $f:[a, b] \longrightarrow \mathbb{R}$ is convex then

$$
f\left(\frac{a+b}{2}\right) \leq \frac{1}{b-a} \int_{a}^{b} f(x) \mathrm{d} x \leq \frac{f(a)+f(b)}{2},
$$

Both inequalities hold in the reversed direction if $f$ is concave.

This inequality received a great deal of attention in the last decade instance, many generalizations and applications being obtained. See [1], [2], [4], [6], [8], [10] and the references therein. Of special interest to us is the following improvements of the Hermite-Hadamard inequality, that can be found in the monograph [7], p. 52:

$$
f\left(\frac{a+b}{2}\right) \leq \frac{1}{2}\left[f\left(\frac{3 a+b}{4}\right)+f\left(\frac{a+3 b}{4}\right)\right] \leq \frac{1}{b-a} \int_{a}^{b} f(x) \mathrm{d} x,
$$

and

$$
\frac{1}{b-a} \int_{a}^{b} f(x) \mathrm{d} x \leq \frac{1}{2}\left[\frac{f(a)+f(b)}{2}+f\left(\frac{a+b}{2}\right)\right] \leq \frac{f(a)+f(b)}{2},
$$

The purpose of the present paper is to establish new Hermite-Hadamard type inequalities within Riemann-Liouville fractional calculus. Unlike the classical case, the functions under attention are not assumed convex or concave, but this fact is asked for the absolute value

Received November 18, 2013, accepted December 23, 2013.

2010 Mathematics Subject Classification. 26A51.

Key words and phrases. Convex function, Hermite-Hadamard inequality, Riemann-Liouville fractional integrals. 
of their derivatives. Under these circumstances we will prove the existence of two strings of inequalities refining the inequalities $(\mathrm{LHH})$ and $(\mathrm{RHH})$.

In what follows we will consider only real-valued functions defined on intervals $[a, b]$ with $0 \leq a<b$, and $n$ is an odd number.

Let $f \in L^{1}[a, b]$ be an integrable function. The Riemann-Liouville integrals $J_{a+}^{\alpha} f$ and $J_{b-}^{\alpha} f$, of order $\alpha>0$, attached to $f$ are defined respectively by

and

$$
J_{a+}^{\alpha} f(x)=\frac{1}{\Gamma(\alpha)} \int_{a}^{x}(x-t)^{\alpha-1} f(t) \mathrm{d} t, \quad \text { for } x>a,
$$

$$
J_{b-}^{\alpha} f(x)=\frac{1}{\Gamma(\alpha)} \int_{x}^{b}(t-x)^{\alpha-1} f(t) \mathrm{d} t, \quad \text { for } x<b .
$$

Here, $\Gamma(\alpha)=\int_{0}^{\infty} e^{-t} t^{\alpha-1} \mathrm{~d} t$ is the Gamma function. We make the convention

$$
J_{a+}^{0} f(x)=J_{b-}^{0} f(x)=f(x) .
$$

The theory of Riemann-Liouville fractional integrals can be found in the book [3].

\section{Main results}

As above, we assume that $[a, b]$ is a compact subinterval of $[0, \infty)$ and $f:[a, b] \rightarrow \mathbb{R}$ is an integrable function.

We define the cumulative to the left $(\alpha, n)$-gap of $f$ by the formula

$$
\begin{aligned}
\mathscr{L}_{\alpha, n}(a, b)= & \sum_{k=0}^{(n-1) / 2} 2 f\left(\frac{a(n-2 k)+b(2 k+1)}{n+1}\right) \\
& -\Gamma(\alpha+1)\left(\frac{n+1}{b-a}\right)^{\alpha} \sum_{k=0}^{\alpha(n-1) / 2}\left[J_{\frac{a(n-2 k)+b(2 k+1)}{n+1}-}^{\alpha} f\left(\frac{a(n-2 k+1)+b \cdot 2 k}{n+1}\right)\right. \\
& \left.+J_{\frac{a(n-2 k-1)+b(2 k+2)}{n+1}-}^{\alpha} f\left(\frac{a(n-2 k)+b(2 k+1)}{n+1}\right)\right] .
\end{aligned}
$$

In the particular case where $\alpha=1$ and $n=3$ we have

$$
\frac{\mathscr{L}_{1,3}(a, b)}{4}=\frac{1}{2}\left[f\left(\frac{3 a+b}{4}\right)+f\left(\frac{a+3 b}{4}\right)\right]-\frac{1}{b-a} \int_{a}^{b} f(t) \mathrm{d} t,
$$

so the cumulative to the left gap $\mathscr{L}_{1,3}(a, b)$ estimates the precision of the right hand side inequality in $(\mathrm{LHH})$.

$$
\frac{1}{b-a} \int_{a}^{b} f(x) \mathrm{d} x \geq \frac{1}{2}\left[f\left(\frac{3 a+b}{4}\right)+f\left(\frac{a+3 b}{4}\right)\right] .
$$


The cumulative to the left gaps $\mathscr{L}_{\alpha, n}(a, b)$ have the same meaning, relative to higher order refinements of $(\mathrm{LHH})$.

The following technical lemma provides a suitable formula for estimating $\mathscr{L}_{\alpha, n}(a, b)$ in absolute value:

Lemma 1. We have

$$
\begin{aligned}
& \mathscr{L}_{\alpha, n}(a, b)=\frac{b-a}{n+1} \\
& \quad \times \sum_{k=0}^{(n-1) / 2}\left[\int_{0}^{1} t^{\alpha} f^{\prime}\left(t \frac{a(n-2 k)+b(2 k+1)}{n+1}+(1-t) \frac{a(n-2 k+1)+b \cdot 2 k}{n+1}\right) \mathrm{d} t\right. \\
& \left.\quad+\int_{0}^{1}\left(t^{\alpha}-1\right) f^{\prime}\left(t \frac{a(n-2 k-1)+b(2 k+2)}{n+1}+(1-t) \frac{a(n-2 k)+b(2 k+1)}{n+1}\right) \mathrm{d} t\right] .
\end{aligned}
$$

Proof. Put

$$
I_{1 k}=\int_{0}^{1} t^{\alpha} f^{\prime}\left(t \frac{a(n-2 k)+b(2 k+1)}{n+1}+(1-t) \frac{a(n-2 k+1)+b \cdot 2 k}{n+1}\right) \mathrm{d} t,
$$

and

$$
I_{2 k}=\int_{0}^{1}\left(t^{\alpha}-1\right) f^{\prime}\left(t \frac{a(n-2 k-1)+b(2 k+2)}{n+1}+(1-t) \frac{a(n-2 k)+b(2 k+1)}{n+1}\right) \mathrm{d} t .
$$

By using the integration by parts and the substitutions

and

$$
u=t \frac{a(n-2 k)+b(2 k+1)}{n+1}+(1-t) \frac{a(n-2 k+1)+b \cdot 2 k}{n+1},
$$

$$
v=t \frac{a(n-2 k-1)+b(2 k+2)}{n+1}+(1-t) \frac{a(n-2 k)+b(2 k+1)}{n+1},
$$

we infer that

$$
\begin{aligned}
I_{1 k}+I_{2 k}= & \frac{2(n+1)}{b-a} f\left(\frac{a(n-2 k)+b(2 k+1)}{n+1}\right)-\Gamma(\alpha+1) \cdot\left(\frac{n+1}{b-a}\right)^{\alpha+1} \\
& \times\left[J_{\frac{a(n-2 k)+b(2 k+1)}{n+1}-}^{\alpha} f\left(\frac{a(n-2 k+1)+b \cdot 2 k}{n+1}\right)\right. \\
& \left.+J_{\frac{a(n-2 k-1)+b(2 k+2)}{n+1}}^{\alpha} f\left(\frac{a(n-2 k)+b(2 k+1)}{n+1}\right)\right]
\end{aligned}
$$

The proof is completed.

We are now in a position to state and prove the following result:

Theorem 1. Assume that $f:[a, b] \rightarrow \mathbb{R}$ is a differentiable function such that $\left|f^{\prime}\right|$ is convex on $[a, b]$. Then

$$
\left|\mathscr{L}_{\alpha, n}(a, b)\right| \leq \frac{b-a}{n+1} \sum_{k=0}^{(n-1) / 2}\left[\frac{\alpha^{2}+5 \alpha+2}{2(\alpha+1)(\alpha+2)}\left|f^{\prime}\left(\frac{a(n-2 k)+b(2 k+1)}{n+1}\right)\right|\right.
$$




$$
\begin{aligned}
& +\frac{1}{(\alpha+1)(\alpha+2)}\left|f^{\prime}\left(\frac{a(n-2 k+1)+b \cdot 2 k}{n+1}\right)\right| \\
& \left.+\frac{\alpha}{2(\alpha+2)}\left|f^{\prime}\left(\frac{a(n-2 k-1)+b(2 k+2)}{n+1}\right)\right|\right] .
\end{aligned}
$$

Proof. Using Lemma 1 and the convexity of $\left|f^{\prime}\right|$ we obtain

$$
\begin{aligned}
\left|\mathscr{L}_{\alpha, n}(a, b)\right| \leq & \frac{b-a}{n+1} \sum_{k=0}^{(n-1) / 2}\left[\left|f^{\prime}\left(\frac{a(n-2 k)+b(2 k+1)}{n+1}\right)\right| \int_{0}^{1} t^{\alpha+1} \mathrm{~d} t\right. \\
& +\left|f^{\prime}\left(\frac{a(n-2 k+1)+b \cdot 2 k}{n+1}\right)\right| \int_{0}^{1} t^{\alpha}(1-t) \mathrm{d} t \\
& +\left|f^{\prime}\left(\frac{a(n-2 k-1)+b(2 k+2)}{n+1}\right)\right| \int_{0}^{1} t\left(1-t^{\alpha}\right) \mathrm{d} t \\
& \left.+\left|f^{\prime}\left(\frac{a(n-2 k)+b(2 k+1)}{n+1}\right)\right| \int_{0}^{1}\left(1-t^{\alpha}\right)(1-t) \mathrm{d} t\right] .
\end{aligned}
$$

The proof ends after a straightforward computation in the right hand side term.

The Beta function is defined by the formula

$$
\mathrm{B}(x, y)=\int_{0}^{1} t^{x-1}(1-t)^{y-1} \mathrm{~d} t \quad \text { for } x, y>0 .
$$

Our next result is as follows:

Theorem 2. Assume that $f:[a, b] \rightarrow \mathbb{R}$ is a differentiable function such that $\left|f^{\prime}\right|^{q}$ is convex on $[a, b]$ for some exponent $q>1$. Then

$$
\begin{aligned}
\left|\mathscr{L}_{\alpha, n}(a, b)\right| \leq & \frac{b-a}{2^{\frac{1}{q}}(n+1)} \sum_{k=0}^{(n-1) / 2}\left\{\left(\frac{1}{\alpha p+1}\right)^{\frac{1}{p}}\right. \\
& \times\left[\left|f^{\prime}\left(\frac{a(n-2 k)+b(2 k+1)}{n+1}\right)\right|^{q}+\left|f^{\prime}\left(\frac{a(n-2 k+1)+b \cdot 2 k}{n+1}\right)\right|^{q}\right]^{\frac{1}{q}} \\
& +\left[\frac{1}{\alpha} \mathrm{B}\left(p+1, \frac{1}{\alpha}\right)\right]^{\frac{1}{p}} \\
& \left.\times\left[\left|f^{\prime}\left(\frac{a(n-2 k-1)+b(2 k+2)}{n+1}\right)\right|^{q}+\left|f^{\prime}\left(\frac{a(n-2 k)+b(2 k+1)}{n+1}\right)\right|^{q}\right]^{\frac{1}{q}}\right\} .
\end{aligned}
$$

where $\frac{1}{p}+\frac{1}{q}=1$.

Proof. According to Lemma 1 and Hölder's inequality, we have

$$
\left|\mathscr{L}_{\alpha, n}(a, b)\right| \leq \frac{b-a}{n+1} \sum_{k=0}^{(n-1) / 2}\left[\left(\int_{0}^{1}\left(t^{\alpha}\right)^{p} \mathrm{~d} t\right)^{\frac{1}{p}}\right.
$$




$$
\begin{aligned}
& \times\left(\int_{0}^{1}\left|f^{\prime}\left(t \frac{a(n-2 k)+b(2 k+1)}{n+1}+(1-t) \frac{a(n-2 k+1)+b \cdot 2 k}{n+1}\right)\right|^{q} \mathrm{~d} t\right)^{\frac{1}{q}} \\
& +\left(\int_{0}^{1}\left(1-t^{\alpha}\right)^{p} \mathrm{~d} t\right)^{\frac{1}{p}} \\
& \left.\times\left(\int_{0}^{1}\left|f^{\prime}\left(t \frac{a(n-2 k-1)+b(2 k+2)}{n+1}+(1-t) \frac{a(n-2 k)+b(2 k+1)}{n+1}\right)\right|^{q} \mathrm{~d} t\right)^{\frac{1}{q}}\right] .
\end{aligned}
$$

Since $|f|^{q}$ is convex on $[a, b]$, we have:

$$
\begin{aligned}
& \int_{0}^{1}\left|f^{\prime}\left(t \frac{a(n-2 k)+b(2 k+1)}{n+1}+(1-t) \frac{a(n-2 k+1)+b \cdot 2 k}{n+1}\right)\right|^{q} \mathrm{~d} t \\
& \quad \leq \frac{1}{2}\left[\left|f^{\prime}\left(\frac{a(n-2 k)+b(2 k+1)}{n+1}\right)\right|^{q}+\left|f^{\prime}\left(\frac{a(n-2 k+1)+b \cdot 2 k}{n+1}\right)\right|^{q}\right]
\end{aligned}
$$

and

$$
\begin{aligned}
& \int_{0}^{1}\left|f^{\prime}\left(t \frac{a(n-2 k-1)+b(2 k+2)}{n+1}+(1-t) \frac{a(n-2 k)+b(2 k+1)}{n+1}\right)\right|^{q} \mathrm{~d} t \\
& \quad \leq \frac{1}{2}\left[\left|f^{\prime}\left(\frac{a(n-2 k-1)+b(2 k+2)}{n+1}\right)\right|^{q}+\left|f^{\prime}\left(\frac{a(n-2 k)+b(2 k+1)}{n+1}\right)\right|^{q}\right] .
\end{aligned}
$$

A simple computation shows that

$$
\int_{0}^{1} t^{\alpha p} \mathrm{~d} t=\frac{1}{\alpha p+1}, \int_{0}^{1}\left(1-t^{\alpha}\right)^{p} \mathrm{~d} t=\frac{1}{\alpha} \mathrm{B}\left(p+1, \frac{1}{\alpha}\right)
$$

and the proof is completed.

Theorem 3. Assume that $f:[a, b] \rightarrow \mathbb{R}$ is a differentiable function such that $\left|f^{\prime}\right|^{q}$ is convex on $[a, b]$ for some exponent $q \geq 1$. Then the following inequality holds:

$$
\begin{aligned}
& \left|\mathscr{L}_{\alpha, n}(a, b)\right| \leq \frac{b-a}{(n+1)(\alpha+1)^{\frac{1}{p}}(\alpha+2)^{\frac{1}{q}}} \\
& \quad \times \sum_{k=0}^{(n-1) / 2}\left\{\left[\left|f^{\prime}\left(\frac{a(n-2 k)+b(2 k+1)}{n+1}\right)\right|^{q}+\frac{1}{\alpha+1}\left|f^{\prime}\left(\frac{a(n-2 k+1)+b \cdot 2 k}{n+1}\right)\right|^{q}\right]^{\frac{1}{q}}\right. \\
& \left.+\frac{\alpha}{2^{\frac{1}{q}}}\left[\left|f^{\prime}\left(\frac{a(n-2 k-1)+b(2 k+2)}{n+1}\right)\right|^{q}+\frac{\alpha+3}{\alpha+1}\left|f^{\prime}\left(\frac{a(n-2 k)+b(2 k+1)}{n+1}\right)\right|^{q}\right]^{\frac{1}{q}}\right\},
\end{aligned}
$$

where $\frac{1}{p}+\frac{1}{q}=1$.

Proof. Using Lemma 1 and the power mean inequality, we have

$$
\begin{aligned}
& \left|\mathscr{L}_{\alpha, n}(a, b)\right| \leq \frac{b-a}{n+1} \sum_{k=0}^{(n-1) / 2}\left[\left(\int_{0}^{1} t^{\alpha} \mathrm{d} t\right)^{\frac{1}{p}}\right. \\
& \quad \times\left(\int_{0}^{1} t^{\alpha}\left|f^{\prime}\left(t \frac{a(n-2 k)+b(2 k+1)}{n+1}+(1-t) \frac{a(n-2 k+1)+b \cdot 2 k}{n+1}\right)\right|^{q} \mathrm{~d} t\right)^{\frac{1}{q}}
\end{aligned}
$$




$$
\begin{aligned}
& +\left(\int_{0}^{1}\left(1-t^{\alpha}\right) \mathrm{d} t\right)^{\frac{1}{p}} \\
& \left.\times\left(\int_{0}^{1}\left(1-t^{\alpha}\right)\left|f^{\prime}\left(t \frac{a(n-2 k-1)+b(2 k+2)}{n+1}+(1-t) \frac{a(n-2 k)+b(2 k+1)}{n+1}\right)\right|^{q} \mathrm{~d} t\right)^{\frac{1}{q}}\right] .
\end{aligned}
$$

Since $|f|^{q}$ is convex on $[a, b]$, we have:

$$
\begin{aligned}
\int_{0}^{1} t^{\alpha} & \left|f^{\prime}\left(t \frac{a(n-2 k)+b(2 k+1)}{n+1}+(1-t) \frac{a(n-2 k+1)+b \cdot 2 k}{n+1}\right)\right|^{q} \mathrm{~d} t \\
\leq & \left.\frac{1}{\alpha+2}\left|f^{\prime}\left(\frac{a(n-2 k)+b(2 k+1)}{n+1}\right)\right|\right|^{q} \\
& +\frac{1}{(\alpha+1)(\alpha+2)}\left|f^{\prime}\left(\frac{a(n-2 k+1)+b \cdot 2 k}{n+1}\right)\right|^{q}
\end{aligned}
$$

and

$$
\begin{aligned}
& \int_{0}^{1}\left(1-t^{\alpha}\right)\left|f^{\prime}\left(t \frac{a(n-2 k-1)+b(2 k+2)}{n+1}+(1-t) \frac{a(n-2 k)+b(2 k+1)}{n+1}\right)\right|^{q} \mathrm{~d} t \\
& \leq \frac{\alpha}{2(\alpha+2)}\left|f^{\prime}\left(\frac{a(n-2 k-1)+b(2 k+2)}{n+1}\right)\right|^{q} \\
&+\frac{\alpha^{2}+3 \alpha}{2(\alpha+1)(\alpha+2)}\left|f^{\prime}\left(\frac{a(n-2 k)+b(2 k+1)}{n+1}\right)\right|^{q} .
\end{aligned}
$$

This completes the proof of the theorem.

Theorem 4. Assume that $f:[a, b] \rightarrow \mathbb{R}$ is a differentiable function such that $\left|f^{\prime}\right|^{q}$ is concave on $[a, b]$ for some exponent $q>1$. Then

$$
\begin{aligned}
\left|\mathscr{L}_{\alpha, n}(a, b)\right| \leq & \frac{b-a}{n+1} \sum_{k=0}^{(n-1) / 2}\left[\left(\frac{1}{\alpha p+1}\right)^{1 / p}\left|f^{\prime}\left(\frac{a(2 n-4 k+1)+b(4 k+1)}{2(n+1)}\right)\right|\right. \\
& \left.+\left(\frac{1}{\alpha} \mathrm{B}\left(p+1, \frac{1}{\alpha}\right)\right)^{1 / p}\left|f^{\prime}\left(\frac{a(2 n-4 k-1)+b(4 k+3)}{2(n+1)}\right)\right|\right]
\end{aligned}
$$

where $\frac{1}{p}+\frac{1}{q}=1$.

Proof. From Lemma 1 and Hölder's integral inequality for $q>1$ and $p=\frac{q}{q-1}$, we have

$$
\begin{aligned}
& \left|\mathscr{L}_{\alpha, n}(a, b)\right| \leq \frac{b-a}{n+1} \sum_{k=0}^{(n-1) / 2}\left[\left(\int_{0}^{1}\left(t^{\alpha}\right)^{p} \mathrm{~d} t\right)^{\frac{1}{p}}\right. \\
& \quad \times\left(\int_{0}^{1}\left|f^{\prime}\left(t \frac{a(n-2 k)+b(2 k+1)}{n+1}+(1-t) \frac{a(n-2 k+1)+b \cdot 2 k}{n+1}\right)\right|^{q} \mathrm{~d} t\right)^{\frac{1}{q}} \\
& \quad+\left(\int_{0}^{1}\left(1-t^{\alpha}\right)^{p} \mathrm{~d} t\right)^{p} \\
& \left.\quad \times\left(\int_{0}^{1}\left|f^{\prime}\left(t \frac{a(n-2 k-1)+b(2 k+2)}{n+1}+(1-t) \frac{a(n-2 k)+b(2 k+1)}{n+1}\right)\right|^{q} \mathrm{~d} t\right)^{\frac{1}{q}}\right] .
\end{aligned}
$$


Since $\left|f^{\prime}\right|^{q}$ is concave on $[a, b]$, we infer from Jensen's inequality for concave functions that

$$
\begin{aligned}
& \int_{0}^{1}\left|f^{\prime}\left(t \frac{a(n-2 k)+b(2 k+1)}{n+1}+(1-t) \frac{a(n-2 k+1)+b \cdot 2 k}{n+1}\right)\right|^{q} \mathrm{~d} t \\
& \leq\left|f^{\prime}\left(\frac{\left.\frac{a(n-2 k)+b(2 k+1)}{n+1}+\frac{a(n-2 k+1)+b \cdot 2 k}{n+1}\right)\left.\right|^{q}}{2}\right)\right| \\
& =\left|f^{\prime}\left(\frac{a(2 n-4 k+1)+b(4 k+1)}{2(n+1)}\right)\right|^{q} .
\end{aligned}
$$

In the same manner,

$$
\begin{aligned}
& \int_{0}^{1}\left|f^{\prime}\left(t \frac{a(n-2 k-1)+b(2 k+2)}{n+1}+(1-t) \frac{a(n-2 k)+b(2 k+1)}{n+1}\right)\right|^{q} \mathrm{~d} t \\
& \quad \leq\left|f^{\prime}\left(\frac{a(2 n-4 k-1)+b(4 k+3)}{2(n+1)}\right)\right|^{q} .
\end{aligned}
$$

Using

$$
\int_{0}^{1} t^{\alpha p} \mathrm{~d} t=\frac{1}{\alpha p+1}, \int_{0}^{1}\left(1-t^{\alpha}\right)^{p} \mathrm{~d} t=\frac{1}{\alpha} \mathrm{B}\left(p+1, \frac{1}{\alpha}\right),
$$

we complete the proof.

Our next result is as follows:

Theorem 5. Assume that $f:[a, b] \rightarrow \mathbb{R}$ is a differentiable function such that $\left|f^{\prime}\right|$ is concave on $[a, b]$ for some exponent $q>1$. Then

$$
\begin{aligned}
& \left|\mathscr{L}_{\alpha, n}(a, b)\right| \leq \frac{b-a}{(\alpha+1)(n+1)} \\
& \quad \times \sum_{k=0}^{(n-1) / 2}\left[\left|f^{\prime}\left(\frac{\alpha+1}{\alpha+2} \cdot \frac{a(n-2 k)+b(2 k+1)}{(n+1)}+\frac{1}{\alpha+2} \cdot \frac{a(n-2 k+1)+b \cdot 2 k}{(n+1)}\right)\right|\right. \\
& \left.\quad+\alpha\left|f^{\prime}\left(\frac{\alpha+1}{2(\alpha+2)} \cdot \frac{a(n-2 k-1)+b(2 k+2)}{(n+1)}+\frac{\alpha+3}{2(\alpha+2)} \cdot \frac{a(n-2 k)+b(2 k+1)}{(n+1)}\right)\right|\right] .
\end{aligned}
$$

Proof. From Lemma 1 we have

$$
\begin{aligned}
& \left|\mathscr{L}_{\alpha, n}(a, b)\right| \leq \frac{b-a}{n+1} \\
& \quad \times \sum_{k=0}^{(n-1) / 2}\left[\int_{0}^{1} t^{\alpha}\left|f^{\prime}\left(t \frac{a(n-2 k)+b(2 k+1)}{n+1}+(1-t) \frac{a(n-2 k+1)+b \cdot 2 k}{n+1}\right)\right| \mathrm{d} t\right. \\
& \left.\quad+\int_{0}^{1}\left(1-t^{\alpha}\right)\left|f^{\prime}\left(t \frac{a(n-2 k-1)+b(2 k+2)}{n+1}+(1-t) \frac{a(n-2 k)+b(2 k+1)}{n+1}\right)\right| \mathrm{d} t\right] .
\end{aligned}
$$

Since $\left|f^{\prime}\right|$ is concave, by Jensen's inequality we obtain

$$
\left|\mathscr{L}_{\alpha, n}(a, b)\right| \leq \frac{b-a}{n+1} \sum_{k=0}^{(n-1) / 2}\left[\left(\int_{0}^{1} t^{\alpha} \mathrm{d} t\right) I_{1}+\left(\int_{0}^{1}\left(1-t^{\alpha}\right) \mathrm{d} t\right) I_{2}\right],
$$


where

$$
\begin{aligned}
I_{1} & =\left|f^{\prime}\left(\frac{\int_{0}^{1} t^{\alpha}\left(t \frac{a(n-2 k)+b(2 k+1)}{n+1}+(1-t) \frac{a(n-2 k+1)+b \cdot 2 k}{n+1}\right) \mathrm{d} t}{\int_{0}^{1} t^{\alpha} \mathrm{d} t}\right)\right| \\
& =\left|f^{\prime}\left(\frac{\alpha+1}{\alpha+2} \cdot \frac{a(n-2 k)+b(2 k+1)}{n+1}+\frac{1}{\alpha+2} \cdot \frac{a(n-2 k+1)+b \cdot 2 k)}{n+1}\right)\right|
\end{aligned}
$$

and

$$
\begin{aligned}
I_{2} & =\left|f^{\prime}\left(\frac{\int_{0}^{1}\left(1-t^{\alpha}\right)\left(t \frac{a(n-2 k-1)+b(2 k+2)}{n+1}+(1-t) \frac{a(n-2 k)+b(2 k+1)}{n+1}\right) \mathrm{d} t}{\int_{0}^{1}\left(1-t^{\alpha}\right) \mathrm{d} t}\right)\right| \\
& =\left|f^{\prime}\left(\frac{\alpha+1}{2(\alpha+2)} \cdot \frac{a(n-2 k-1)+b(2 k+2)}{n+1}+\frac{\alpha+3}{2(\alpha+2)} \cdot \frac{a(n-2 k)+b(2 k+1)}{n+1}\right)\right|
\end{aligned}
$$

and the proof is completed.

Remark 1. For $\alpha=1$ in the Theorems 1,2, 3, 4 respectively 5, we recover the results stated in ([9, Theorems 6-10]). Also, for $\alpha=1$ in Lemma 1, we get ([9, Lemma 1]).

Remark 2. For $n=3$ in the Theorems 1, 2, 3, respectively 4 , we recover the results stated in ([5, Theorems 1-4]). Also for $\alpha=1$ in Lemma 1, we get ([5, Lemma 1]).

We end our paper by considering the cumulative to the right $(\alpha, n)-$ gap defined by the formula

$$
\begin{aligned}
\mathscr{R}_{\alpha, n}(a, b)= & -\sum_{k=0}^{(n-1) / 2}\left[f\left(\frac{a(n-2 k+1)+b \cdot 2 k}{n+1}\right)+f\left(\frac{a(n-2 k-1)+b(2 k+2)}{n+1}\right)\right] \\
& +\Gamma(\alpha+1)\left(\frac{n+1}{b-a}\right) \sum_{k=0}^{\alpha(n-1) / 2}\left[J_{\frac{a(n-2 k+1)+b \cdot 2 k}{n+1}+}^{\alpha} f\left(\frac{a(n-2 k)+b(2 k+1)}{n+1}\right)\right. \\
& \left.+J_{\frac{a(n-2 k)+b(2 k+1)}{n+1}+}^{\alpha} f\left(\frac{a(n-2 k-1)+b(2 k+2)}{n+1}\right)\right],
\end{aligned}
$$

In the particular case where $\alpha=1$ and $n=3$ we have

$$
\frac{\mathscr{R}_{1,3}(a, b)}{4}=-\frac{1}{2}\left[\frac{f(a)+f(b)}{2}+f\left(\frac{a+b}{2}\right)\right]+\frac{1}{b-a} \int_{a}^{b} f(t) \mathrm{d} t,
$$

so the cumulative to the right gap $\mathscr{R}_{1,3}(a, b)$ estimates the precision of the left hand side inequality in $(R H H)$,

$$
\frac{1}{b-a} \int_{a}^{b} f(x) \mathrm{d} x \leq \frac{1}{2}\left[\frac{f(a)+f(b)}{2}+f\left(\frac{a+b}{2}\right)\right]
$$

Using the above techniques, one can prove companions of all the results we proved for the cumulative to the left $(\alpha, n)$-gap. The starting point is the following formula for comput$\operatorname{ing} \mathscr{R}_{\alpha, n}(a, b)$. 
Lemma 2. We have

$$
\begin{aligned}
& \mathscr{R}_{\alpha, n}(a, b)=\frac{b-a}{n+1} \\
& \quad \times \sum_{k=0}^{(n-1) / 2}\left[\int_{0}^{1} t^{\alpha} f^{\prime}\left(t \frac{a(n-2 k+1)+b \cdot 2 k}{n+1}+(1-t) \frac{a(n-2 k)+b(2 k+1)}{n+1}\right) \mathrm{d} t\right. \\
& \left.\quad+\int_{0}^{1}\left(t^{\alpha}-1\right) f^{\prime}\left(t \frac{a(n-2 k)+b(2 k+1)}{n+1}+(1-t) \frac{a(n-2 k-1)+b(2 k+2)}{n+1}\right) \mathrm{d} t\right] .
\end{aligned}
$$

Using Lemma 2, one can prove various estimates of $\mathscr{R}_{\alpha, n}(a, b)$ such as

$$
\begin{aligned}
\left|\mathscr{R}_{\alpha, n}(a, b)\right| \leq & \frac{b-a}{n+1} \sum_{k=0}^{(n-1) / 2}\left[\frac{1}{\alpha+2}\left|f^{\prime}\left(\frac{a(n-2 k+1)+b \cdot 2 k}{n+1}\right)\right|\right. \\
& +\frac{\alpha^{2}+\alpha+2}{2(\alpha+1)(\alpha+2)}\left|f^{\prime}\left(\frac{a(n-2 k)+b(2 k+1)}{n+1}\right)\right| \\
& \left.+\frac{\alpha^{2}+3 \alpha}{2(\alpha+1)(\alpha+2)}\left|f^{\prime}\left(\frac{a(n-2 k-1)+b(2 k+2)}{n+1}\right)\right|\right] .
\end{aligned}
$$

\section{References}

[1] M. Bessenyei, The Hermite-Hadamard Inequality on Simplices, American Mathematical Monthly, 115 (2008), 339-345.

[2] S. S. Dragomir and C. E. M. Pearce, Selected Topic on Hermite-Hadamard Inequalities and Applications, Melbourne and Adelaide, December, 2000.

[3] R. Gorenflo and F. Mainardi, Fractional Calculus: Integral and Differential Equations of Fractional Order, Springer Verlag, Wien, 1997.

[4] H. Kavurmaci and M. Avci, M. E. Özdemir, New inequalities of Hermite-Hadamard type for convex functions with applications, arXiv: 1006.1593v1.

[5] M. Mihai, Some Hermite-Hadamard type inequalities obtained via Riemann-Liouville fractional calculus. (submitted).

[6] F.-C. Mitroi and C. I. Spiridon, Hermite-Hadamard type inequalities of convex functions with respect to a pair of quasi-arithmetic means, Math. Rep., 14 (2012).

[7] C. P. Niculescu and L.-E. Persson, Convex Functions and their Applications. A Contemporary Approach, CMS Books in Mathematics vol. 23, Springer-Verlag, New York, 2006.

[8] C. P. Niculescu, The Hermite-Hadamard inequality for log-convex functions, Nonlinear Analysis 75 (2012), 662-669.

[9] M. Emin Özdemir, A. Ekinci and A. Akdemir, Some new integral inequalities for functions whose derivatives of absolute values are convex and concave, RGMIA Research Report Collection, 15 (2012), Article 48, 12 pp.

[10] S. Wassowicz and A. Witkowski, On some inequality of Hermite-Hadamard type, Opuscula Math., 32(2012), 591-600.

Department of Mathematics, University of Craiova, Street A. I. Cuza 13, Craiova, RO-200585, Romania.

E-mail: marcelamihai58@yahoo.com

Department of Mathematics, University of Craiova, Street A. I. Cuza 13, Craiova, RO-200585, Romania.

E-mail: dan_alexion@yahoo.com 